\title{
Fifteen Limit Cycles Bifurcating from a Perturbed Cubic Center
}

\author{
Amor Menaceur, ${ }^{1}$ Mufda Alrawashdeh, ${ }^{2}$ Sahar Ahmed Idris $\mathbb{D}^{3},{ }^{3,4}$ and Hala Abd-Elmageed ${ }^{5}$ \\ ${ }^{1}$ Laboratory of Analysis and Control of Differential Equations ACED, Department of Mathematics, University of Guelma, \\ P.O. Box 401, Guelma 24000, Algeria \\ ${ }^{2}$ Department of Mathematics, College of Sciences and Arts, ArRas, Qassim University, Buraydah, Saudi Arabia \\ ${ }^{3}$ College of Industrial Engineering, King Khalid University, Abha, Saudi Arabia \\ ${ }^{4}$ Department of Mathematics, College of Sciences, Juba University, Juba, Sudan \\ ${ }^{5}$ Department of Mathematics, Faculty of Science, SVU, Qena 83523, Egypt
}

Correspondence should be addressed to Sahar Ahmed Idris; sa6044690@gmail.com

Received 19 October 2021; Accepted 12 November 2021; Published 1 December 2021

Academic Editor: Sundarapandian Vaidyanathan

Copyright (c) 2021 Amor Menaceur et al. This is an open access article distributed under the Creative Commons Attribution License, which permits unrestricted use, distribution, and reproduction in any medium, provided the original work is properly cited.

In this work, we study the bifurcation of limit cycles from the period annulus surrounding the origin of a class of cubic polynomial differential systems; when they are perturbed inside the class of all polynomial differential systems of degree six, we obtain at most fifteenth limit cycles by using the averaging theory of first order.

\section{Introduction and Statement of the Main Result}

Hilbert in 1900 was interested in the maximum number of the limit cycles that a polynomial differential system of a given degree can have. This problem is the well-known 16th Hilbert problem, which together with the Riemann conjecture are the two problems of the famous list of 23 problems of Hilbert which remain open. See for more details $[1,2]$.

A classical way to produce limit cycles is by perturbing a system which has a center, in such a way that limit cycles bifurcate in the perturbed system from some of the periodic orbits of the period annulus of the center of the unperturbed system [3-7].

In [8], the authors improved the result of the maximum number of limit cycles for a class of polynomial differential systems which bifurcate from the period annulus surrounding the origin of the system:

$$
\left\{\begin{array}{l}
\dot{u}=v-v(u-y+a)(u+v+a), \\
\dot{v}=-u+u(u-v+a)(u+v+a),
\end{array}\right.
$$

where $(u-y+a)(u+v+a)=1$ is a conic, $a^{2} \neq 1$, and $|a| \leq \sqrt{2}$ by using the first order of the averaging theory method.

In [9], the authors improved the result of the maximum number of limit cycles of sixth polynomial differential systems which bifurcate from the period annulus surrounding the origin of the system:

$$
\left\{\begin{array}{l}
\dot{u}=-v\left(u-v^{2}-a\right)^{2}, \\
\dot{v}=u\left(u-v^{2}-a\right)^{2}
\end{array}\right.
$$

where $u-v^{2}-a=0$ is a conic and $a \neq 0$, by using the first order of the averaging theory method.

In this work, we perturb the cubic systems equation (1). Thus, we consider these classes of all polynomial differential systems of degree $n$, i.e.,

$$
\left\{\begin{array}{l}
\dot{u}=v-v(u-y+a)(u+v+a)+\varepsilon P(u, v), \\
\dot{v}=-u+u(u-v+a)(u+v+a)+\varepsilon Q(u, v),
\end{array}\right.
$$

where $(u-v+a)(u+v+a)=1$ is a conic, $|a|>\sqrt{2}, P(u, v)$ and $Q(u, v)$ are the real polynomials of degree $n \geq 3$, and $\varepsilon$ is 
a small parameter. Main result of this study is the following Theorem 1.

Theorem 1. For the sufficiently small $|\varepsilon|$ and the polynomials $P(u, v)$ and $Q(u, v)$ having degree 6 , suppose that $|a|>\sqrt{2}$, system equation (3) has at most 15 limit cycles bifurcating from the period annulus surrounding the origin of cubic polynomial differential system equation (1) using averaging theory of first order (Figures 1 and 2).

\section{The Averaging Theory of First Order}

Theorem 2. Consider the following two initial value problems:

$$
\dot{x}=\varepsilon R(t, x)+\varepsilon^{2} G(t, x, \varepsilon), x(0)=x_{0},
$$

and

$$
\dot{y}=\varepsilon f^{0}(y), y(0)=x_{0},
$$

where $x, y$, and $x_{0} \in D$ is an open domain of $\mathbb{R}, t \in[0, \infty)$, $\varepsilon \in\left(0, \varepsilon_{0}\right], R$ and $G$ are the periodic functions with their period $T$ with its variable $t$, and $f^{0}(y)$ is the average function of $R(t, y)$ with respect to $t$, i.e.,

$$
f^{0}(y)=\frac{1}{T} \int_{0}^{T} F(t, y) d t
$$

\section{Assume that}

(i) $R, \partial R / \partial x, \partial^{2} R / \partial x^{2}, G$, and $\partial G / \partial x$ are well defined, continuous, and bounded by a constant independent by $\varepsilon \in\left(0, \varepsilon_{0}\right]$ in $[0, \infty) \times D$

(ii) $T$ is a constant independent of $\varepsilon$

(iii) $y(t)$ belongs to $D$ on the time scale $1 / \varepsilon$. Then, the following statements hold. (a) On the time scale $1 / \varepsilon$, we have

$$
x(t)-y(t)=O(\varepsilon), \text { as } \varepsilon \longrightarrow 0 .
$$

(b) If $p$ is an equilibrium point of the averaged system equation (5), such that

$$
\left.\frac{\partial f^{0}}{\partial y}\right|_{y=p} \neq 0
$$

Then, system equation (4) has a $T$-periodic solution $\phi(t, \varepsilon) \longrightarrow p$ as $\varepsilon \longrightarrow 0$.

(c) If equation (8) is a negative, the corresponding periodic solution $\phi(t, \varepsilon)$ of equation (4) according to $(t, x)$ is asymptotically stable for all $\varepsilon$ sufficiently small, and if equation (8) is a positive, then it is unstable.

For more details on the averaging method, see $[10,11]$.

\section{Proof of Theorem 1}

For $|a|>2$, the cubic system equation (1) has a unique period annulus:

$$
A=\left\{(u, v): 0<u^{2}+v^{2}<\frac{a^{2}-2}{2}\right\} .
$$

According to Figures 1 and 2, this proof is based on the first order of the averaging theory method, in polar coordinates $(r, \theta)$, where $u=r \cos \theta, v=r \sin \theta$, and $r>0$. We take

$$
P(u, v)=\sum_{k=1}^{n} \sum_{i+j=k} p_{i j} u^{i} v^{j}, Q(u, v)=\sum_{k=1}^{n} \sum_{i+j=k} q_{i j} u^{i} v^{j} .
$$

Equation (3) can be written as follows:

$$
\left\{\begin{array}{l}
\dot{r}=\varepsilon \sum_{k=1}^{n}\left(\cos \theta M_{k}(\cos \theta, \sin \theta)+\sin \theta N_{k}(\cos \theta, \sin \theta)\right) r^{k} \\
\dot{\theta}=S(r, \theta)+\varepsilon \sum_{k=1}^{n}\left(\cos \theta N_{k}(\cos \theta, \sin \theta)-\sin \theta M_{k}(\cos \theta, \sin \theta)\right) r^{k-1},
\end{array}\right.
$$

where

$$
\begin{aligned}
\frac{d r}{d \theta}= & \varepsilon \sum_{k=1}^{n} \cos \theta M_{k}(\cos \theta, \sin \theta)+\sin \theta N_{k}(\cos \theta, \sin \theta) \\
& \cdot \frac{r^{k}}{S(r, \theta)}+\varepsilon^{2} R(r, \theta, \varepsilon) .
\end{aligned}
$$$$
\begin{aligned}
& M_{k}(\cos \theta, \sin \theta)=\sum_{i+j=k} p_{i j}^{i} \cos ^{i} \theta \sin ^{j} \theta, \\
& N_{k}(\cos x, \sin x)=\sum_{i+j=k} q_{i j} \cos ^{i} \theta \sin ^{j} \theta,
\end{aligned}
$$

and

The averaged function of equation (14) is

$$
f^{0}(r)=\frac{1}{2 \pi} \sum_{k=1}^{n} r^{k}
$$

$$
S(r, \theta)=(r(\cos \theta-\sin \theta)+a)(r(\cos \theta+\sin \theta)+a)-1 .
$$

$$
\cdot \int_{0}^{2 \pi} \frac{\cos \theta M_{k}(\cos \theta, \sin \theta)+\sin \theta N_{k}(\cos \theta, \sin \theta)}{S(r, \theta)} d \theta \text {. }
$$

Therefore, we have 


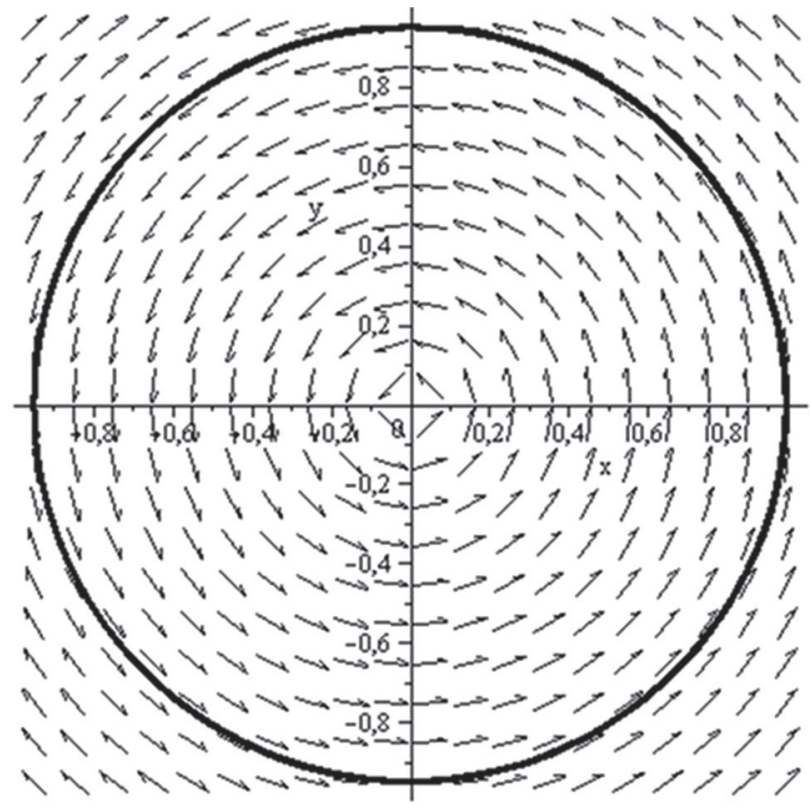

FIGURE 1: Phase portrait of the cubic system equation (1) with $a=2$.

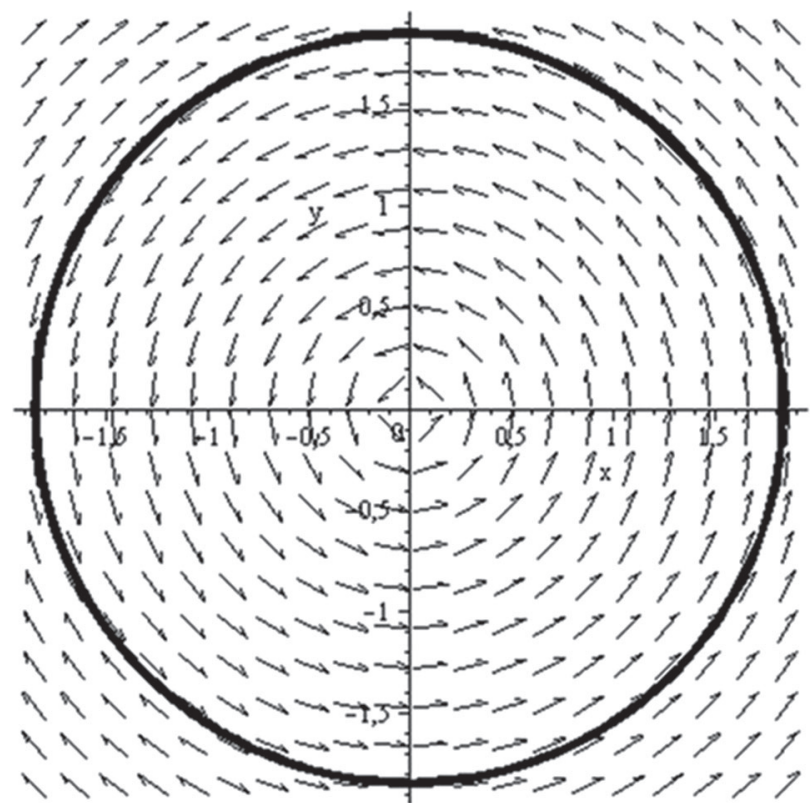

Figure 2: Phase portrait of the cubic system equation (1) with $a=2$.

For $n=6$, we get

$$
f^{0}(r)=\sum_{k=1}^{6} \sum_{i+j=k}^{6}\left(p_{i j} A_{i+1, j}(r)+q_{i j} A_{i, j+1}(r)\right) r^{k}
$$

where

$$
A_{p, q}=\frac{1}{2 \pi} \int_{0}^{2 \pi} \frac{\cos ^{p} \theta \sin ^{q} \theta}{S(r, \theta)} d \theta
$$

According to Theorem 2, every simple zero of the average function $f^{0}(r)$ provides a limit cycle of system equation (3). Now, we prove Theorem 1; in the first step, we compute the integral $f^{0}(r)$, and in the second step, the number of its simple zeros is studied.

Lemma 1. From the above, we have

$$
A_{0,0}=-\frac{G_{1}-G_{2}}{2 S H_{1} H_{2}} \text { and } A_{1,0}=\frac{a\left(G_{1}-G_{2}\right)}{4 r S H_{1} H_{2}}+\frac{G_{1}+G_{2}}{4 r H_{1} H_{2}} \text {, }
$$

where 


$$
\begin{aligned}
& H_{1}=\sqrt{(a-1)^{2}-r^{2}}, \\
& H_{2}=\sqrt{(a+1)^{2}-r^{2}}, \\
& G_{1}=\sqrt{-2 r^{2}-2 a S+2}, \\
& G_{2}=\sqrt{-2 r^{2}+2 a S+2},
\end{aligned}
$$

$$
\underset{0}{2 \pi} \frac{\cos \theta d \theta}{S(r, \theta)} \equiv \frac{1}{i r^{2}} C \frac{z^{2}+1}{\left(z-z_{1}\right)\left(z-z_{2}\right)\left(z-z_{3}\right)\left(z-z_{4}\right)} d z,
$$

whose poles are

$$
z_{1,2}=\frac{-a-\sqrt{2 r^{2}-a^{2}+2} \pm \sqrt{-2 r^{2}+2+2 a \sqrt{2 r^{2}-a^{2}+2}}}{2 r}
$$

with

$$
S=\sqrt{2 r^{2}-a^{2}+2} \neq 0 .
$$

Proof. Assume that $z=e^{i \theta}$ and $C$ is the circle $|z|=1$; we get $2 \pi \frac{d \theta}{S(r, \theta)} \equiv \frac{2}{i r^{2} C} \frac{z}{\left(z-z_{1}\right)\left(z-z_{2}\right)\left(z-z_{3}\right)\left(z-z_{4}\right)} d z$,

By applying the residue theorem, for $|a|>\sqrt{2}$, we obtain $\left|z_{1}\right|<1, \mid z_{3}<1, C$ encloses the two singular points of the integrand, so

$$
\begin{aligned}
A_{0,0} & =\frac{1}{2 \pi}^{0} \frac{d \theta}{S(r, \theta)}, \\
& =\frac{1}{\pi i r^{2}} \frac{z}{\left(z-z_{1}\right)\left(z-z_{2}\right)\left(z-z_{3}\right)\left(z-z_{4}\right)} d z \\
& =\frac{2}{r^{2}}\left(\frac{z_{1}}{\left(z_{1}-z_{2}\right)\left(z_{1}-z_{3}\right)\left(z_{1}-z_{4}\right)}+\frac{z_{3}}{\left(z_{3}-z_{1}\right)\left(z_{3}-z_{2}\right)\left(z_{3}-z_{4}\right)}\right) .
\end{aligned}
$$

Therefore, we have

$$
\begin{aligned}
A_{0,0} & =-\frac{\sqrt{-2 r^{2}-2 a \sqrt{2 r^{2}-a^{2}+2}+2}-\sqrt{-2 r^{2}+2 a \sqrt{2 r^{2}-a^{2}+2}+2}}{2 \sqrt{2 r^{2}-a^{2}+2} \sqrt{(a-1)^{2}-r^{2}} \sqrt{(a+1)^{2}-r^{2}}}, \\
A_{1,0} & =\frac{1}{2 \pi}{ }^{0} \frac{{ }^{0}}{\cos \theta d \theta} \\
& =\frac{1}{2 \pi i r^{2}} \frac{z^{2}+1}{\left(z-z_{1}\right)\left(z-z_{2}\right)\left(z-z_{3}\right)\left(z-z_{4}\right)} d z, \\
& =\frac{1}{r^{2}}\left(\frac{z_{1}^{2}+1}{\left(z_{1}-z_{2}\right)\left(z_{1}-z_{3}\right)\left(z_{1}-z_{4}\right)}+\frac{z_{3}^{2}+1}{\left(z_{3}-z_{1}\right)\left(z_{3}-z_{2}\right)\left(z_{3}-z_{4}\right)}\right) .
\end{aligned}
$$


Therefore, we get

$$
\begin{gathered}
A_{1,0}=\frac{a\left(\sqrt{-2 r^{2}-2 a \sqrt{2 r^{2}-a^{2}+2}+2}-\sqrt{-2 r^{2}+2 a \sqrt{2 r^{2}-a^{2}+2}+2}\right)}{4 r \sqrt{2 r^{2}+2-a^{2}} \sqrt{(a-1)^{2}-r^{2}} \sqrt{(a+1)^{2}-r^{2}}} \\
+\frac{\sqrt{-2 r^{2}-2 a \sqrt{2 r^{2}-a^{2}+2}+2}+\sqrt{-2 r^{2}+2 a \sqrt{2 r^{2}-a^{2}+2}+2}}{4 r \sqrt{(a-1)^{2}-r^{2}} \sqrt{(a+1)^{2}-r^{2}}} .
\end{gathered}
$$

This completes the proof.

Lemma 2. Under the previous notations, we have

(a) $A_{2 k+1,0}=-(a / r) A_{2 k, 0}+\left(r^{2}+1-a^{2} / 2 r^{2}\right) A_{2 k-1,0}$

(b) $A_{2 k, 0}=-(a / r) A_{2 k-1,0}+\left(r^{2}+1-\right.$

$\left.a^{2} / 2 r^{2}\right) A_{2 k-2,0}+\left(\lambda_{k-1} / 2^{k}(k-1) ! r^{2}\right)$,

Where $\lambda_{k-1}=3.5 \ldots(2 k-3)$

(c) $A_{0,2 k}=\sum_{i=0}^{k}(-1)^{i} C_{k}^{i} A_{2 i, 0}$

(d) $A_{n-2 m+1,2 m}=\sum_{i=0}^{m}(-1)^{i} C_{m}^{i} A_{n-2 m+2 i+1,0}, \quad m=1, \ldots,[n$ $+1 / 2$ ].

Proof. Putting $u=r \cos \theta, v=r \sin \theta$, we get for (a) and (b)

$$
\begin{aligned}
\frac{\cos ^{n} \theta}{S(r, \theta)} & =\frac{\cos ^{n} \theta}{\left(a^{2}-2 r^{2}-2 / 2\right)+2(r \cos \theta+(a / 2))^{2}}, \\
& =\frac{-2 a r \cos ^{n-1} \theta+\left(r^{2}+1-a^{2}\right) \cos ^{n-2} \theta+S(r, \theta) \cos ^{n-2} \theta}{2 r^{2} S(r, \theta)}, \\
& =-\frac{a}{r} \frac{\cos ^{n-1} \theta}{S(r, \theta)}+\frac{r^{2}+1-a^{2}}{2 r^{2}} \frac{\cos ^{n-2} \theta}{S(r, \theta)}+\frac{1}{2 r^{2}} \cos ^{n-2} \theta .
\end{aligned}
$$

Thus,

$$
\begin{aligned}
A_{n, 0}= & -\frac{a}{2 \pi r} \int_{0}^{2 \pi} \frac{\cos ^{n-1} \theta}{S(r, \theta)} d \theta+\frac{r^{2}+1-a^{2}}{4 \pi r^{2}} \int_{0}^{2 \pi} \frac{\cos ^{n-2} \theta}{S(r, \theta)} d \theta \\
& +\frac{1}{4 \pi r^{2}} \int_{0}^{2 \pi} \cos ^{n-2} \theta d \theta .
\end{aligned}
$$

Therefore,

$$
\begin{aligned}
A_{2 k+1,0} & =-\frac{a}{r} A_{2 k, 0}+\frac{r^{2}+1-a^{2}}{2 r^{2}} A_{2 k-1,0}, \\
A_{2 k, 0} & =-\frac{a}{r} A_{2 k-1,0}+\frac{r^{2}+1-a^{2}}{2 r^{2}} A_{2 k-2,0}+\frac{1}{4 \pi r^{2}} \int_{0}^{2 \pi} \cos ^{2 k-2} \theta d \theta,
\end{aligned}
$$

and

$$
\int_{0}^{2 \pi} \cos ^{2(k-1)} \theta d \theta=\frac{\pi \lambda_{k-1}}{2^{k-2}(k-1) !}
$$

where $\lambda_{k-1}=3.5 \ldots(2 k-3), \lambda_{k}=(2 k-1) \lambda_{k-1}([12])$. Thus,

$$
\begin{gathered}
A_{2 k, 0}=-\frac{a}{r} A_{2 k-1,0}+\frac{r^{2}+1-a^{2}}{2 r^{2}} A_{2 k-2,0}+\frac{\lambda_{k-1}}{2^{k}(k-1) ! r^{2}}, \\
\frac{\sin ^{2 k} \theta}{S(r, \theta)}=\frac{\left(1-\cos ^{2} \theta\right)^{k}}{S(r, \theta)} \\
=\sum_{i=0}^{k}(-1)^{i} C_{k}^{i} \frac{\cos ^{2 i} \theta}{S(r, \theta)},
\end{gathered}
$$

then

$$
A_{0,2 k}=\sum_{i=0}^{k}(-1)^{i} C_{k}^{i} A_{2 i, 0},
$$

(d)

$$
\begin{aligned}
\frac{\cos ^{n-2 m+1} \theta \sin ^{2 m} \theta}{S(r, \theta)}= & \frac{\cos ^{n-2 m+1} \theta\left(1-\cos ^{2} \theta\right)^{m}}{S(r, \theta)} \\
= & \sum_{i=0}^{m}(-1)^{i} C_{m}^{i} \frac{\cos ^{n-2 m+2 i+1} \theta}{S(r, \theta)}, \\
& m=1, \ldots,\left[\frac{n+1}{2}\right] .
\end{aligned}
$$

Thus,

$$
A_{n-2 m+1,2 m}=\sum_{i=0}^{m}(-1)^{i} C_{m}^{i} A_{n-2 m+2 i+1,0}, m=1, \ldots,\left[\frac{n+1}{2}\right] .
$$

This completes the proof.

Remark 1. $A_{p, 2 k+1}=0$.

By Lemmas 1 and 2, we have

$$
\begin{aligned}
A_{2,0}= & \frac{1}{4 \pi r^{2}} \int_{0}^{2 \pi} d \theta-\frac{a}{r} A_{1,0}+\frac{r^{2}+1-a^{2}}{2 r^{2}} A_{0,0} \\
= & \frac{1}{2 r^{2}}-\frac{a}{r}\left(\frac{a\left(G_{1}-G_{2}\right)}{4 r S H_{1} H_{2}}+\frac{G_{1}+G_{2}}{4 r H_{1} H_{2}}\right) \\
& +\frac{r^{2}+1-a^{2}}{2 r^{2}}\left(-\frac{G_{1}-G_{2}}{2 S H_{1} H_{2}}\right) \\
= & -\frac{1}{4 S H_{1} H_{2} r^{2}}\left(-2 S H_{1} H_{2}+a S\left(G_{1}+G_{2}\right)\right. \\
& \left.+\left(r^{2}+1\right)\left(G_{1}-G_{2}\right)\right) .
\end{aligned}
$$


Then,

$$
\begin{aligned}
A_{0,2}= & A_{0,0}-A_{2,0} \\
& =-\frac{G_{1}-G_{2}}{2 S H_{1} H_{2}}-\left(-\frac{1}{4 S H_{1} H_{2} r^{2}}\left(-2 S H_{1} H_{2}+a S\left(G_{1}+G_{2}\right)+\left(r^{2}+1\right)\left(G_{1}-G_{2}\right)\right)\right) \\
& =\frac{-2 S H_{1} H_{2}+a S\left(G_{1}+G_{2}\right)-\left(r^{2}-1\right)\left(G_{1}-G_{2}\right)}{4 S H_{1} H_{2} r^{2}},
\end{aligned}
$$

and we also have

$$
\begin{aligned}
A_{3,0}= & \frac{-4 a S H_{1} H_{2}+\left(r^{2}+a^{2}+1\right) S\left(G_{1}+G_{2}\right)+a\left(3 r^{2}-a^{2}+3\right)\left(G_{1}-G_{2}\right)}{8 S H_{1} H_{2} r^{3}}, \\
A_{1,2}= & -\frac{\left(-4 a S H_{1} H_{2}+\left(-r^{2}+a^{2}+1\right) S\left(G_{1}+G_{2}\right)+a\left(r^{2}-a^{2}+3\right)\left(G_{1}-G_{2}\right)\right)}{8 S H_{1} H_{2} r^{3}}, \\
A_{4,0}= & -\frac{1}{8 S H_{1} H_{2} r^{4}}\left[\left(-4 r^{2}-2\left(a^{2}+1\right)\right) S H_{1} H_{2}+2 a\left(r^{2}+1\right) S\left(G_{1}+G_{2}\right)\right. \\
& \left.+\left(r^{4}+2\left(a^{2}+1\right) r^{2}+\left(-a^{4}+2 a^{2}+1\right)\right)\left(G_{1}-G_{2}\right)\right] \\
A_{2,2}= & \frac{1}{8 S H_{1} H_{2} r^{4}}\left[2\left(-a^{2}-1\right) S H_{1} H_{2}+2 a S\left(G_{1}+G_{2}\right)\right. \\
& \left.+\left(-r^{4}+2 a^{2} r^{2}+\left(-a^{4}+2 a^{2}+1\right)\right)\left(G_{1}-G_{2}\right)\right]
\end{aligned}
$$

and

$$
\begin{aligned}
A_{0,4}= & -\frac{1}{8 S H_{1} H_{2} r^{4}}\left[2\left(2 r^{2}-a^{2}-1\right) S H_{1} H_{2}+2 a\left(-r^{2}+1\right) S\left(G_{1}+G_{2}\right)\right. \\
& \left.+\left(r^{4}+2\left(a^{2}-1\right) r^{2}+\left(-a^{4}+2 a^{2}+1\right)\right)\left(G_{1}-G_{2}\right)\right], \\
A_{5,0}= & \frac{1}{16 S H_{1} H_{2} r^{5}}\left[4 a\left(-3 r^{2}-2\right) S H_{1} H_{2}+\left(r^{4}+2\left(2 a^{2}+1\right) r^{2}+\left(-a^{4}+4 a^{2}+1\right)\right) S\left(G_{1}+G_{2}\right)\right. \\
& \left.+a\left(5 r^{4}+10 r^{2}-a^{4}+5\right)\left(G_{1}-G_{2}\right)\right], \\
A_{3,2}= & -\frac{1}{16 S H_{1} H_{2} r^{5}}\left[4 a\left(-r^{2}-2\right) S H_{1} H_{2}+\left(-r^{4}+2 a^{2} r^{2}+\left(-a^{4}+4 a^{2}+1\right)\right) S\left(G_{1}+G_{2}\right)\right. \\
& \left.+\left(-a r^{4}+2 a\left(a^{2}+2\right) r^{2}+a\left(-a^{4}+5\right)\right)\left(G_{1}-G_{2}\right)\right] \\
A_{1,4}= & \frac{1}{16 S H_{1} H_{2} r^{5}}\left[4 a\left(r^{2}-2\right) S H_{1} H_{2}+\left(r^{4}-2 r^{2}+\left(-a^{4}+4 a^{2}+1\right)\right) S\left(G_{1}+G_{2}\right)\right. \\
& \left.+\left(-3 a r^{4}+2 a\left(2 a^{2}-1\right) r^{2}+a\left(5-a^{4}\right)\right)\left(G_{1}-G_{2}\right)\right],
\end{aligned}
$$




$$
\begin{aligned}
A_{6,0}= & -\frac{1}{16 S H_{1} H_{2} r^{6}}\left[\left(-7 r^{4}+2\left(-5 a^{2}-3\right) r^{2}+2\left(a^{4}-4 a^{2}-1\right)\right) S H_{1} H_{2}\right. \\
& +a\left(3 r^{4}+2\left(a^{2}+3\right) r^{2}+\left(-a^{4}+2 a^{2}+3\right)\right) S\left(G_{1}+G_{2}\right) \\
& \left.+\left(r^{6}+3\left(2 a^{2}+1\right) r^{4}+3\left(4 a^{2}-a^{4}+1\right) r^{2}+\left(6 a^{2}-3 a^{4}+1\right)\right)\left(G_{1}-G_{2}\right)\right], \\
A_{4,2}= & \frac{1}{16 S H_{1} H_{2} r^{6}}\left[\left(r^{4}+\left(-6 a^{2}-2\right) r^{2}+\left(2 a^{4}-8 a^{2}-2\right)\right) S H_{1} H_{2}\right. \\
& +\left((-a) r^{4}+\left(2 a^{3}+2 a\right) r^{2}+\left(2 a^{3}-a^{5}+3 a\right)\right) S\left(G_{1}+G_{2}\right) \\
& \left.+\left(-r^{6}+\left(2 a^{2}-1\right) r^{4}+\left(8 a^{2}-a^{4}+1\right) r^{2}+\left(6 a^{2}-3 a^{4}+1\right)\right)\left(G_{1}-G_{2}\right)\right] .
\end{aligned}
$$

In addition, we have

$$
\begin{aligned}
& A_{2,4}=-\frac{1}{16 S H_{1} H_{2} r^{6}}\left[\left(r^{4}+2\left(1-a^{2}\right) r^{2}+2\left(a^{4}-4 a^{2}-1\right)\right) S H_{1} H_{2}\right. \\
& +\left(-a r^{4}+2 a\left(a^{2}-1\right) r^{2}+a\left(-a^{4}+2 a^{2}+3\right)\right) S\left(G_{1}+G_{2}\right) \\
& \left.+\left(r^{6}+\left(-2 a^{2}-1\right) r^{4}+\left(a^{4}+4 a^{2}-1\right) r^{2}+\left(6 a^{2}-3 a^{4}+1\right)\right)\left(G_{1}-G_{2}\right)\right], \\
& A_{0,6}=\frac{1}{16 S H_{1} H_{2} r^{6}}\left[\left(-7 r^{4}+2\left(a^{2}+3\right) r^{2}+2\left(a^{4}-4 a^{2}-1\right)\right) S H_{1} H_{2}\right. \\
& +\left(3 a r^{4}+2 a\left(a^{2}-3\right) r^{2}+a\left(-a^{4}+2 a^{2}+3\right)\right) S\left(G_{1}+G_{2}\right) \\
& \left.+\left(-r^{6}+3\left(-2 a^{2}+1\right) r^{4}+3\left(a^{4}-1\right) r^{2}+\left(-3 a^{4}+6 a^{2}+1\right)\right)\left(G_{1}-G_{2}\right)\right], \\
& A_{7,0}=\frac{1}{32 S H_{1} H_{2} r^{7}}\left[\left(4(-3 a-7) r^{4}+4\left(7 a-10 a^{2}-8\right) r^{2}+8\left(a^{4}-4 a^{2}+3 a-1\right)\right) S H_{1} H_{2}\right. \\
& +\left(r^{6}+\left(4 a^{2}+12 a-1\right) r^{4}+\left(-a^{4}+8 a^{3}-8 a^{2}+24 a-5\right) r^{2}\right. \\
& \left.+\left(-4 a^{5}+3 a^{4}+8 a^{3}-12 a^{2}+12 a-3\right)\right) S\left(G_{1}+G_{2}\right) \\
& +\left((5 a+4) r^{6}+\left(24 a^{2}-5 a+12\right) r^{4}+\left(-a^{5}-12 a^{4}+48 a^{2}-25 a+12\right) r^{2}\right. \\
& \left.\left.+\left(3 a^{5}-12 a^{4}+24 a^{2}-15 a+4\right)\right)\left(G_{1}-G_{2}\right)\right] \\
& A_{5,2}=-\frac{1}{32 S H_{1} H_{2} r^{7}}\left[\left(4(3 a-7) r^{4}+4\left(11 a-10 a^{2}-8\right) r^{2}+8\left(a^{4}-4 a^{2}+3 a-1\right)\right) S H_{1} H_{2}\right. \\
& +\left(-r^{6}+\left(-4 a^{2}+12 a-5\right) r^{4}+\left(a^{4}+8 a^{3}-16 a^{2}+24 a-7\right) r^{2}\right. \\
& \left.+\left(-4 a^{5}+3 a^{4}+8 a^{3}-12 a^{2}+12 a-3\right)\right) S\left(G_{1}+G_{2}\right) \\
& +\left((-5 a+4) r^{6}+\left(24 a^{2}-25 a+12\right) r^{4}+\left(a^{5}-12 a^{4}+48 a^{2}-35 a+12\right) r^{2}\right. \\
& \left.\left.+\left(3 a^{5}-12 a^{4}+24 a^{2}-15 a+4\right)\right)\left(G_{1}-G_{2}\right)\right]
\end{aligned}
$$




$$
\begin{aligned}
& A_{3,4}=\frac{1}{32 S H_{1} H_{2} r^{7}}\left[\left(4(5 a-7) r^{4}+4\left(15 a-10 a^{2}-7\right) r^{2}+8\left(a^{4}-4 a^{2}+3 a-1\right)\right) S H_{1} H_{2}\right. \\
& +\left(r^{6}+\left(-8 a^{2}+12 a-5\right) r^{4}+\left(3 a^{4}+8 a^{3}-24 a^{2}+24 a-9\right) r^{2}\right. \\
& \left.+\left(-4 a^{5}+3 a^{4}+8 a^{3}-12 a^{2}+12 a-3\right)\right) S\left(G_{1}+G_{2}\right) \\
& +\left((-3 a+4) r^{6}+\left(-4 a^{3}+24 a^{2}-33 a+12\right) r^{4}\right. \\
& +\left(3 a^{5}-12 a^{4}+48 a^{2}-45 a+12\right) r^{2} \\
& \left.\left.+\left(3 a^{5}-12 a^{4}+24 a^{2}-15 a+4\right)\right)\left(G_{1}-G_{2}\right)\right], \\
& A_{1,6}=-\frac{1}{32 S H_{1} H_{2} r^{7}}\left[\left(4(3 a-7) r^{4}+4\left(19 a-10 a^{2}-7\right) r^{2}+8\left(a^{4}-4 a^{2}+3 a-1\right)\right) S H_{1} H_{2}\right. \\
& +\left(-r^{6}+\left(-8 a^{2}+12 a-1\right) r^{4}+\left(5 a^{4}+8 a^{3}-32 a^{2}+24 a-11\right) r^{2}\right. \\
& \left.+\left(-4 a^{5}+3 a^{4}+8 a^{3}-12 a^{2}+12 a-3\right)\right) S\left(G_{1}+G_{2}\right) \\
& +\left((3 a+4) r^{6}+\left(-12 a^{3}+24 a^{2}-29 a+12\right) r^{4}\right. \\
& +\left(5 a^{5}-12 a^{4}+48 a^{2} .-55 a+12\right) r^{2} \\
& \left.\left.+\left(3 a^{5}-12 a^{4}+24 a^{2}-15 a+4\right)\right)\left(G_{1}-G_{2}\right)\right] .
\end{aligned}
$$

Using equation (15), we get

$f^{0}(r)=\frac{1}{r S H_{1} H_{2}}\left(X S H_{1} H_{2}+Y S\left(G_{1}+G_{2}\right)+Z\left(G_{1}-G_{2}\right)\right)$,

where

$$
X=\left(x_{4} r^{4}+x_{2} r^{2}+x_{0}\right), Y=\left(y_{6} r^{6}+y_{4} r^{4}+y_{2} r^{2}+y_{0}\right),
$$

and

$$
Z=\left(z_{6} r^{6}+z_{4} r^{4}+z_{2} r^{2}+\frac{\left(a^{2}-1\right) x_{0}+2 a y_{0}}{2}\right),
$$

with the coefficients $x_{i}, y_{i}$, and $z_{i}$ the polynomials in the coefficients of $a, p_{i, j}$, and $q_{i, j}$.

In fact, there are only ten independent parameters between $x_{i}, y_{i}$, and $z_{i}$ with respect to $p_{i j}, q_{i j}$, and $a$. In order to bound the zeros number of numerator of $f^{0}(r)$, it is sufficient to bound the zeros number of

$$
K(r)=X^{2} S^{2} H_{1}^{2} H_{2}^{2}-\left[Y S\left(G_{1}+G_{2}\right)+Z\left(G_{1}-G_{2}\right)\right]^{2} .
$$

Since

$$
\begin{aligned}
\left(G_{1}+G_{2}\right)\left(G_{1}-G_{2}\right) & =-4 a S \\
\left(G_{1}+G_{2}\right)^{2} & =4\left(1-r^{2}\right)+2 G_{1} G_{2}
\end{aligned}
$$

and

$$
\left(G_{1}-G_{2}\right)^{2}=4\left(1-r^{2}\right)-2 G_{1} G_{2}
$$

we have

$$
\begin{aligned}
K(r)= & X^{2} S^{2} H_{1}^{2} H_{2}^{2}-4 Y^{2} S^{2}\left(1-r^{2}\right)-4 Z^{2}\left(1-r^{2}\right) \\
& +8 a Y Z S^{2}-2\left(Y^{2} S^{2}-Z^{2}\right) G_{1} G_{2} .
\end{aligned}
$$

Finally, in order to bound the zeros number of the above expression, we should bound the zeros of the following polynomial:

$$
\begin{aligned}
H(r)= & {\left[X^{2} S^{2} H_{1}^{2} H_{2}^{2}-4 Y^{2} S^{2}\left(1-r^{2}\right)-4 Z^{2}\left(1-r^{2}\right)+8 a Y Z S^{2}\right]^{2} } \\
& -4\left(Y^{2} S^{2}-Z^{2}\right)^{2} G_{1}^{2} G_{2}^{2} .
\end{aligned}
$$

We have 


$$
\begin{aligned}
H(r)= & S^{2}\left[\left(16 Y^{4} a^{2}\right) S^{4}\right. \\
& +\left(\left(4 Y^{2}\left(r^{2}-1\right)+8 Y Z a+X^{2}\left((a-1)^{2}-r^{2}\right)\left((a+1)^{2}-r^{2}\right)\right)^{2}\right. \\
& \left.-4 Y^{4}\left(2 r^{2}-2\right)^{2}-32 Y^{2} Z^{2} a^{2}\right) S^{2} \\
& +\left(16 Z^{4} a^{2}+8 Z^{2}\left(r^{2}-1\right)\left(4 Y^{2}\left(r^{2}-1\right)+8 Y Z a+X^{2}\left((a-1)^{2}-r^{2}\right)\left((a+1)^{2}-r^{2}\right)\right)\right. \\
& \left.\left.+8 Y^{2} Z^{2}\left(2 r^{2}-2\right)^{2}\right)\right] .
\end{aligned}
$$

Therefore, we get

$$
H(r)=S^{2}\left(d_{30} r^{30}+\cdots+d_{2} r^{2}+d_{0}\right),
$$

where $d_{i}$ are the polynomials in $a, x_{i}, y_{i}$, and $z_{i}$. We conclude that $f^{0}(r)$ has at most 15 simple zeros. Hence, Theorem 1 is proved.

\section{Conclusion}

As we know, the limit cycles and a polynomial differential system is the well-known 16th Hilbert problem, which together with the Riemann conjecture are the two problems of the famous list of 23 problems of Hilbert which remain open. In addition, a classical way to produce limit cycles is by perturbing a system which has a center, in such a way that limit cycles bifurcate in the perturbed system from some of the periodic orbits of the period annulus of the center of the unperturbed system; in this work, by using the averaging theory of first order, we study the bifurcation of limit cycles from the period annulus surrounding the origin of a class of cubic polynomial differential systems; when they are perturbed inside the class of all polynomial differential systems of degree six, we have obtained at most 15th limit cycles for this kind of the problem; in the next study, we will try to extend the same tools but for higher degrees.

\section{Data Availability}

No data were used to support the study.

\section{Conflicts of Interest}

The authors declare that they have no conflicts of interest.

\section{Acknowledgments}

The authors extend their appreciation to the Deanship of Scientific Research at King Khalid University for funding this work through Research Group Project (R.G.P-2/53/42).

\section{References}

[1] L. Hongwei, "Limit cycles in a sextic lyapunov system," Nonlinear Dynamics, vol. 72, pp. 555-559, 2013.

[2] C. Hilbert, "Mathematische probleme, lecture, second internat," Nachrichten von der Gesellschaft der Wissenschaften zu Göttingen, Mathematisch-Physikalische Klasse, pp. 253-297, American Mathematical Society, Providence, RI, USA, 1900.
[3] L. Feng and W. Miao, "Bifurcation of limit cycles in a quintic system with ten parameters," Nonlinear Dynamics, vol. 71, pp. 213-222, 2013.

[4] H. Giacomini, J. Llibre, and M. Viano, "On the nonexistence, existence and uniqueness of limit cycles," Nonlinearity, vol. 9, no. 2, pp. 501-516, 1996.

[5] J. Giné and J. Llibre, "Limit cycles of cubic polynomial vector fields via the averaging theory," Nonlinear Analysis: Theory, Methods \& Applications, vol. 66, no. 8, pp. 1707-1721, 2007.

[6] A. Menaceur, S. Boulaaras, S. Alkhalaf, and S. Jain, "Limit cycles of a class of polynomial differential systems bifurcating from the periodic orbits of a linear center," Symmetry, vol. 12, no. 8, p. $15,2020$.

[7] A. Menaceur, S. Boulaaras, A. Makhlouf, K. Rajagobal, and M. Abdalla, "Limit cycles of a class of perturbed differential systems via the first-order averaging method," Complexity, vol. 2021, Article ID 5581423, 6 pages, 2021.

[8] S. Li, Y. Zhao, and J. Li, "On the number of limit cycles of a perturbed cubic polynomial differential center," Journal of Mathematical Analysis and Applications, vol. 404, no. 2, pp. 212-220, 2013.

[9] A. Menaceur and S. Boulaaras, "A number of limit cycle of sextic polynomial differential systems via the averaging theory," Boletim da Sociedade Paranaense de Matemática, vol. 39, no. 4, pp. 181-197, 2021.

[10] B. Coll, A. Gasull, and R. Prohens, "Bifurcation of limit cycles from two families of centers," Dynamics of Continuous, Discrete and Impulsive Systems, vol. 12, pp. 275-288, 2005.

[11] J. Murdock, A. Sanders, and F. Verhultst, "Averaging methods in nonlinear dynamical systems," Applied Mathematical Sciences, vol. 59, 2007.

[12] M. Abramowitz and I. Stegun, "Handbook of mathematical functions with formulas, graphs, and mathematical tables," National Bureau of Standards Applied Mathematics Series, Us Government Printing Office, Washington, DC, USA, 1964. 